\title{
A TESTING METHODOLOGY FOR AN OPEN SOFTWARE E-LEARNING PLATFORM
}

\author{
Ana Cavalli, Stéphane Maag, Sofia Papagiannaki and Georgios Verigakis \\ GET/Institut National des Télécommunications \\ Laboratoire SAMOVAR / UMR CNRS 5157 \\ 9 rue Charles Fourier \\ F-91011 Evry Cedex, France \\ e-mails : \{Ana.Cavalli, Stephane.Maag, Sofia.Papagiannaki,Georgios.Verigakis\}@int-evry.fr
}

\section{Fatiha Zaïdi}

University of Paris Sud 11,

Laboratoire de Recherche en Informatique,

UMR CNRS 8623

Bat. 490 University of Paris Sud

91405 Orsay Cedex, France

e-mail :Fatiha.Zaidi@lri.fr

\begin{abstract}
This paper presents an outline of a methodology for the test of an open software e-learning platform. The methodology includes a test generation method from UML descriptions, in particular Sequence, Activity, Class diagrams and Navigation maps. The studied open software e-learning platform is dotLRN, an open source enterprise-class suite of web applications and portal framework for supporting course management, online communities and collaborations. The work presented in this paper is being developed in the framework of the E-LANE ${ }^{1}$ project, an European and Latin American collaboration for the creation of an open software integrated platform for e-learning.
\end{abstract}

Keywords: UML, dotLRN, XMI, OCL, testing methodology, test generation methods

\section{Introduction}

In the last few years major progress has been achieved in the design of elearning methodologies and platforms, particularly developing tools and methods to facilitate to users the access to these technologies. More recently, this evolution has strengthened the idea to develop open software platforms by 
integrating existing applications already used for e-learning. In this context, educational organizations can organize the teaching of a collection of courses using e-learning technologies, overcoming the geographical barriers, addressing to a larger audience and last but not least, by reducing the costs. To reduce the costs, organizations only need to deploy a powerful web server with the goal that the users may access these services using an open software platform connected to internet. Moreover, these platforms are designed to stimulate users to use and integrate new services.

This paper presents the work being developed in the framework of the European-Latin American New Education (E-LANE) project, an European and Latin American collaboration for the creation of an open software integrated platform for e-learning in which GET/INT is a partner. This project is part of the European Commission program @LIS Demonstration Projects. The base platform for the development ofE-LANE is dotLRN, which is an open software platform composed by a suite of web applications and a portal framework of an e-learning system.

These open software e-learning platforms integrating existing applications developed by different teams need to be tested to insure that these applications are integrated together smoothly and correctly. The integration of new services by users also needs to be checked to guarantee the systems functionalities are preserved. In particular, these systems are exposed to many threats: a web server has to respond to requests from each node in the network. For instance, testing should guarantee that a user cannot gain permissions over the system he is not supposed to have. In addition to this, modern web applications are becoming increasingly complex and mission critical. This situation becomes even worse because of the lack of standardization in the web clients (browsers). Testing has to assert the system usability. For instance, an user is unable to complete a process because the content of the web page does not appear correctly or, due to a slow network connection, he may be unable to login because of an unrealistic timeout value. Our approach takes into account how these functional aspects have been implemented, checking that they conform to the specification.

In this paper, we propose a testing methodology for the dotLRN platform based on a test generation method and present some ideas of how to test new services integrated by the users. We also give a short presentation of a new approach for testing e-learning services, which is complementary of the test generation method. In this new approach, we propose to describe in OCL [9] the expected properties of the web service and to check these properties on the service execution traces.

The methodology presented in this paper is inspired by the work presented in $[1,3]$, which describes how UML diagrams developed in the Analysis phase are analyzed in an automated way to produce test cases. In relation to this work the paper presents several new contributions. First at all, none of these work is well 
adapted to the test of a web application tool. In our work, we need to check the graphical user interfaces but also the content of the generated pages. There is an extension of UML for Web Applications but it is more focused on the Design View. One of the contributions of this paper is to provide an answer to these issues. Therefore, in order to produce diagrams which convey the information needed to feed our tests we considered the work presented in [7] and [6].

Other contributions given by this paper are the following: to present the techniques and tools which are used for the conformance testing of the platform. The methodology we propose includes the following steps: description of the web services in UML, in particular use cases using Activity diagrams, Sequence diagram, Class diagram and Navigation map; export of diagrams into XMI format [10]; parsing of the XMI in order to generate test scripts; generation of tests scripts (using the different languages for scripts). This paper also presents a data modelling approach using UML profiles.

In the following sections the article first describes the dotLRN platform (section 2). Section 3 presents how to specify dotLRN using UML models for testing. In section 4, we describe the tools to automate the test generation from these models. Section 5 introduces an approach for the data modelling and section 6 presents the perspectives for this work. Finally, section 7 gives the conclusion.

\section{The dotLRN e-learning platform}

dotLRN ${ }^{2}$ is an open source web based e-learning platform and portal framework, designed to support course management, online communities and collaboration.

In dotLRN there are three main portal types: user, class and community portals. The user portal is the private space that each user owns, while the class and community portals contain all the pages related to a specific class or community. Each of these three portal types is divided into four sections: the main space, the calendar, the files and the control panel. The pages in dotLRN are composed of portlets. Portlets are small portals that have some specific functionalities, like the forums, the news or the calendar.

A user portal is created automatically whenever a new user is registered in dotLRN, but the class and community portals are created by the site-wide administrator according to the needs of the users. When a new class or community portal is created, the site-wide administrator assigns one or more users as administrators of this portal. For example, for computer science class portal, the administrators can be the professor and his teaching assistants, while for a photography group community portal, the administrator will be one or 
more students. The responsibilities of a portal administrator are to add content, customize the layout and decide the policy of the portal.

When a class or community has an open policy, any user can join, while when the policy is closed only the administrator can add users. A third policy exists, the wait policy, where any user can ask to join and then the administrator will decide to accept or deny this user.

\section{UML models for testing}

In order to derive our test cases, we need to grasp and describe the functionality of the system in a formal way. A model-based approach development of Web applications can be performed using UML notation. We use a design methodology which is based on a UML extension for hypermedia [8]. It consists of three main steps that constitute the conceptual model, the navigation map, and the presentation model.

The conceptual model is built taking into account the functional requirements captured with use cases. We discover the use cases of the system under consideration and document them in a Requirements Document which contains the scope of the system, the main actors, the use case diagram and textual description of each use case [5].

$>$ From this conceptual model, the navigation space model, also represented as a static class model, is constructed. It defines a view on the conceptual model showing which classes of this latter may be visited through navigation in the Web application. Finally, a dynamic presentation model is represented by UML sequence diagrams describing the collaborations and behaviors of the navigational objects and access primitives. In order to specify our model, the following diagrams are involved:

- Class Diagram to introduce the main classes of the system.

- Activity Diagram for each actor to display dependencies among the use cases.

- Navigation Map to provide information about the dynamic content of the web pages.

- Sequence Diagram for each use case describing the main and the alternative scenarios of the use case to represent the dynamic presentation model.

In our lab we develop a tool that implements our methodology and its components are shown in Figure 1. Initially it analyzes the dependencies found in the activity diagrams and combined with the data input generates test sequences. These test sequences are then used in the test execution component, along with the information in the Use Cases and the Navigation Map in order to 


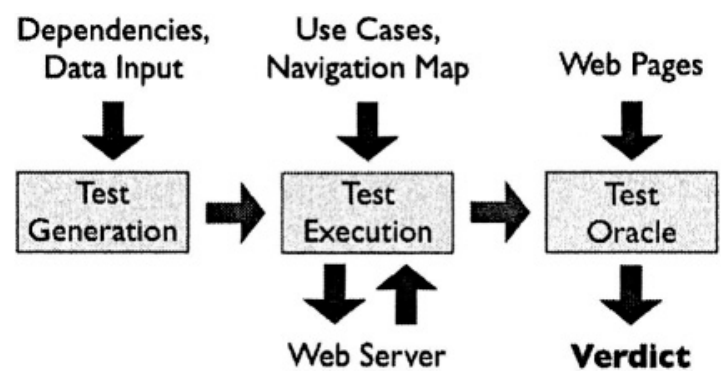

Figure 1. Outline of our methodology

perform the actual requests to the dotLRN server. The last component, parses the response of the server and compares it with the UML specification of the expected web page. Finally, it gives the verdict to show that either the test has executed succesfully, or that it has failed.

\subsection{From Conceptual Model to Navigation Map}

In order to specify the dotLRN platform we first need to build a conceptual model of the application domain taking into account the functional requirements captured with use cases. Techniques such as finding classes and associations, and defining inheritance structures are used.

Then, the Navigation Map of a web application introduced in [7] is used because it provides information about the dynamic content of each web page which is part of the system as well as the links between the different web pages. This information is essential during the parsing of the HTML pages (section 4.3). The Navigation Map is a Class diagram where each web page is a class and a link between two pages is an association between the two respective classes.

\subsection{Modelling Use Case Dependencies}

The use cases of a system are not independent. In order one use case to be executed, another should have taken place before. For instance, in dotLRN the user should login before being able to do anything else. Since the automation of the testing procedure is also of concern, we have to describe somehow these dependencies.

We achieve this by introducing an activity diagram where the vertices represent use cases and edges are sequential dependencies between the use cases. An edge in such a diagram denotes that the use case in the tail has to be executed before the use case in the head. 
In the testing phase, before simulating the scenarios in the Sequence diagrams these activity diagrams should be scanned to obtain the sequence by which the use cases will be tested.

\subsection{Sequence Diagram}

In UML, a Sequence diagram realizes the interaction of objects via the interchange of messages in time. Similarly, as in activity diagrams the objects are instances of a class described in the Class diagram. Usually the sequence diagrams describe a single scenario.

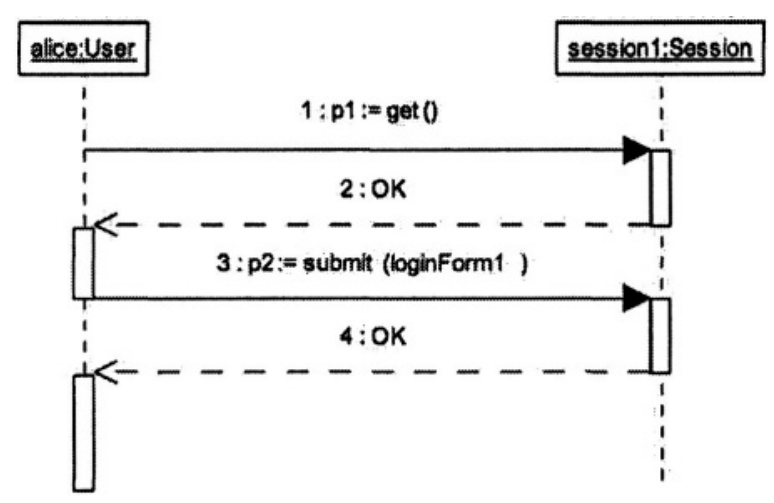

Figure 2. Sequence diagram for "Login" use case

We enumerate the messages as described in [3] so we can illustrate a number of alternative scenarios in the same diagram. According to this convention, capital letters denote alternatives (error messages). By adopting this tactic we can derive easily the different Message_Sequences [1] related to the same use case. Figure 2 shows the respective sequence diagram for the "Login" use case.

Our Sequence diagrams are also parameterized since input parameters can influence the execution and constitute separate Choices [1]. Such a parameter can be the email of an expected User. If this is the case, the dotLRN page is displayed, otherwise a warning appears in the Log In page.

\section{Automating the test generation}

To automate the test generation, our goal is first to parse the UML diagrams obtained from the previous steps. Therefore, based on these diagrams, we generate the necessary requests to the dotLRN server and then check if the server's replies are as expected by the previous models.

Based on these requirements, we had to choose the components that were required to build our test suite. 


\subsection{The programming language}

Since we are dealing with UML, it would be more efficient to choose an object-oriented language. We also wanted this language to provide easy string handling and a high level of abstraction for network communications. The programming language that we found as the most suitable was Python [4]. Python is a modern object-oriented language which combines remarkable power with very clear syntax. Its built-in modules provide numerous functions that facilitate string handling and networking.

\subsection{Parsing and executing the UML}

Although we could use any XML parser to parse the XMI, due to the high complexity of the standard we decided to use a specialized XMI parser. The one we used was the parser included in the System Modelling Workbench tool ${ }^{3}$. It is free, open-source and also is written in Python, making it easier to integrate with our code. Being open-source it also enabled us to fix some incompatibility issues that appeared when used with XMI produced by the Poseidon tool.

\subsection{Parsing the HTML pages}

Since HTML mixes presentation and content data, the HTML output of dotLRN does not allow us to extract the information we want without first looking the implementation details. To avoid this we need to change the page templates of dotLRN in order to provide the data in a more formal way. We achieve this by adding $i d$ attributes to the tags we want to query. For example, to the td tag that contains the user's name in the user pages will have an attribute $i d=$ "username". That way we can query any page independently of the implementation of the layout of the page.

\section{Inserting Test Data into the Model}

In order to execute our tests and observe the behavior of the system it is necessary to substitute the formal parameters by the actual values. For instance in case we want to test whether a user with a valid email and password can $\log$ in successfully in the system, we have to provide to the test a valid pair of email and password. In related literature the tester provides this input through the user interface of the test suite before the execution of each test. In our case since we are interested in automatically creating some scripts which will be immediately executed we would like to integrate somehow this information in our model. 
An approach to the problem of representing data values in UML is the use of a UML Profile. UML Profiles are an extension mechanism provided by UML that enables us to add specific features. UML profiles are implemented using stereotypes and tagged values.

Different solutions could be proposed for the design of a suitable UML Profile, but our solution will focus on simplicity and ease of implementation. When we want to represent an instance $\mathbf{I}$ of a class $\mathbf{C}$, we can define $\mathbf{I}$ as a new class having the same attributes and operations with $\mathbf{C}$. Each attribute of $\mathbf{I}$ will have a tagged value containing the actual value of the respective attribute of $\mathbf{C}$.

This method requires some extra development effort in order to check the correctness of the given values. However, it can be used with older versions of UML that are supported better from most of the currently available open source CASE tools.

\section{Perspectives}

The software tool presented in the previous sessions perform the parsing of the UML specification, the generation and translation of the tests to the XMI form and their execution on the dotLRN platform. The approach that we introduced automatically parses and executes the UML diagrams. However it could be equally possible instead to generate the code that will do the specified test. The generated code could be written in any language and will have the benefit that it can be used to run the tests from any computer, since it will run without the need of our software.

Concerning the test, in a first step, we started with the test of communication interfaces and user requirements. Next steps will be the test of authentication mechanisms and application contents (for instance, to check the content of the required web page). For that purpose, we need to treat data at the specification level. Indeed, to test if a user is permitted to access to the system, we need to check if it enters his proper login and password.

Another issue that we still investigate is related to a new test method. Indeed, we decide to propose another step-wise approach to automate the test generation and execution. This approach has no interaction with the system under test as the test generation method presented in this paper. This new approach is well fitted for service developers that do not intend to spend time at the analysis phase. The approach is based on the verification of service properties directly on the service trace execution. For that purpose, we install a sniffer between the client and the server to obtain dynamically all the $h t m l$ pages of each request on the service. At the specification level, we associate to each class and each operation, properties that have to be verified once the execution of the service is performed. These properties are written using the OCL language and represent the requirements of the web service that we want to test. This language 
is not usable straightforward. We need to transform it in order to check it on the traces. Thus, we translate the OCL described properties in regular expressions. Afterwards, these regular expressions are those that we will check on the real traces execution. At GET/INT, we have developed a tool to perform this verification [2].

This approach using OCL constraints allows to define formally functionalities that services are intended to supply. Furthermore, this solution can also be used for the test of new services. Using this approach, we are able to check if the new added service (i.e. new functionality) works properly according to the OCL defined constraint. Moreover, we are able to test that existing services are preserved, performing regression tests by reusing OCL constraints already checked.

\section{Conclusion}

We have presented in this paper a new approach to test the functionality of an e-learning, web based system, the dotLRN platform. This platform presents the advantage to be an open source toolkit for building scalable, communityoriented web applications.

The method and the software tool we propose has been applied to dotLRN platform but they are generic enough and can be applied to other e-learning and web based systems.

The test method is based on the test of objectives, which are selected taking into account the experts and designers advice. These tests are based on use cases and cover all relevant aspects of the system behavior. Even if we cannot guarantee a total error coverage, it can be guaranteed for the selected tests. We propose a UML models technique for testing, that relies on a design methodology divided in three main steps, i.e. the conceptual model, the navigation model and the presentation model.

\section{References}

[1] F. Basanieri, A. Bertolino, and E. Marchetti. The Cow_suit Approach to Planning and Deriving Test Suites in UML Projects. Proc. Fifth International Conference on the Unified Modeling Language - the Language and its applications UML 2002, LNCS 2460, Dresden, Germany, pages 383-397, September 2002.

[2] Emmanuel Bayse, Ana Cavalli, Manuel Nunez, and Fatiha Zaidi. A passive Testing Approach based on Invariants: Application to the WAP. Computer Networks journal, 2004.

[3] L. C. Briand and Y. Labiche. A UML-Based Approach to System Testing. Software and Systems Modeling, 1(1):10-42, 2002.

[4] Jr. Drake Christopher A. Jones, Fred L. Python \& XML. O'Reilly \& Associates, 1st edition, 2001.

[5] A. Cockburn. Writing Effective Use Cases. Addison-Wesley, 1st edition, 2000. 
[6] J. Conallen. Modelling Web Application Architectures with UML. Communications of the ACM, 42(10):63-70, 1999.

[7] J. Conallen. Building Web Applications With UML. Addison-Wesley, 2nd edition, 2002.

[8] N. Koch, H. Baumeister, R. Hennicker, and L. Mandel. Extending UML to Model Navigation and Presentation in Web applications. Proc. of Modeling Web applications in the UML Workshop, UML2000, 2000.

[9] UML Object Constraint Language (2.0 submission). http://www.omg.org/cgibin/apps/doc?ad/03-01-07.pdf.

[10] XML Metadata Interchange. http://www.omg.org/cgi-bin/apps/doc?formal/03-05-02.pdf. 